\title{
Endoscopic closure of a perforated peptic ulcer
}

A 42-year old woman with Wilson's disease was admitted with a history of acute-onset upper abdominal pain. Physical examination revealed signs of peritonitis in the upper abdomen, and an abdominal CT scan demonstrated a localized pneumoperitoneum ( $\bullet$ Fig. 1).

An esophagogastroduodenoscopy (EGD) (GIF2T60 double-channel endoscope; Olympus, Tokyo, Japan) was performed under general anesthesia using $\mathrm{CO}_{2}$ insufflation, and revealed a clearly demarcated $(2 \times 3 \mathrm{~mm})$ perforated prepyloric ulcer in the anterior wall ( $\bullet$ Fig. 2 ).

The patient's clinical condition permitted an attempt at endoscopic management. To achieve closure, the edges of the ulcer were approximated with a twin grasper (OTSC Twin Grasper; Ovesco Endoscopy AG, Tübingen, Germany) whereupon an over-the-scope clip (OTSC) (Ovesco) was applied ( $\bullet$ Figs. 3 and 4 ).

The procedure was supplemented with intra-abdominal lavage using 2 liters of lukewarm saline instilled through an infraumbilical drain (Blake Silicone Drain; $7 \mathrm{~mm}$ ) and subsequently evacuated. So that it was possible to document the efficacy of the procedure, the patient was given oral methylene blue to drink on the first postoperative day. Since no blue fluid showed up in the drainage, the intra-abdominal drain was removed. On the third postoperative day, oral feeds were started. A follow-up EGD on day 4 demonstrated an intact OTSC closure ( $\bullet$ Fig. 5) and the patient was discharged from the hospital the same day with treatment for Helicobacter pylori eradication.

Biopsies from the ulcer margin showed benign histomorphology. At the followup visit 4 weeks later, the patient reported a completely uneventful recovery with immediate return to work, and an EGD revealed complete mucosal healing with the OTSC still in place.

In summary, this report contains one of the original descriptions of the use of the OTSC system for closure of a perforated peptic ulcer. This technique will have implications for the endoscopic repair of transmural defects in the gastrointestinal tract.

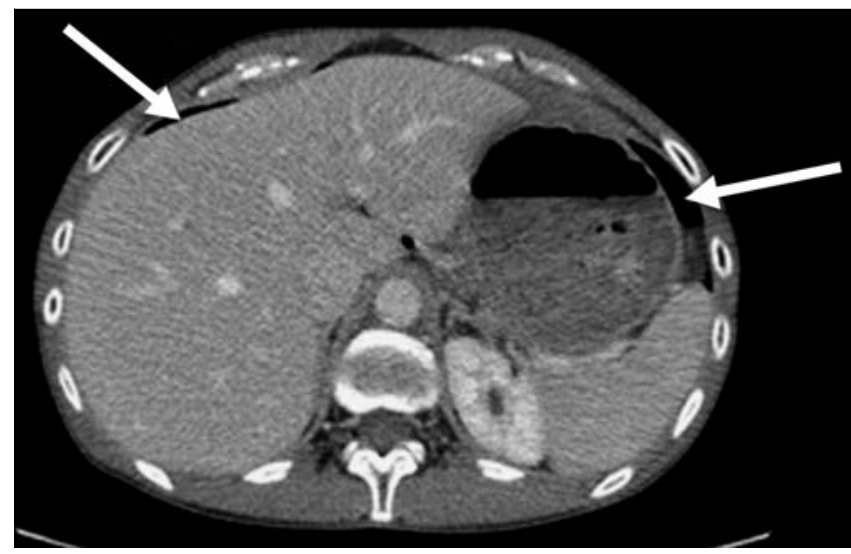

Fig. 1 Abdominal computed tomography (CT) scan at admission of the patient, with arrows indicating pneumoperitoneum.

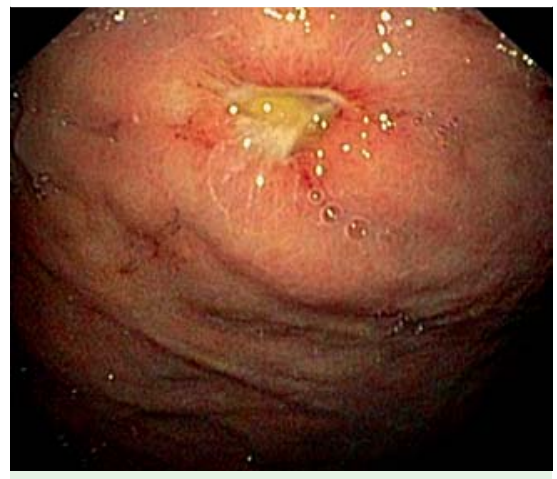

Fig. 2 Prepyloric gastric ulcer located along the anterior wall, covered by a fibrin clot.

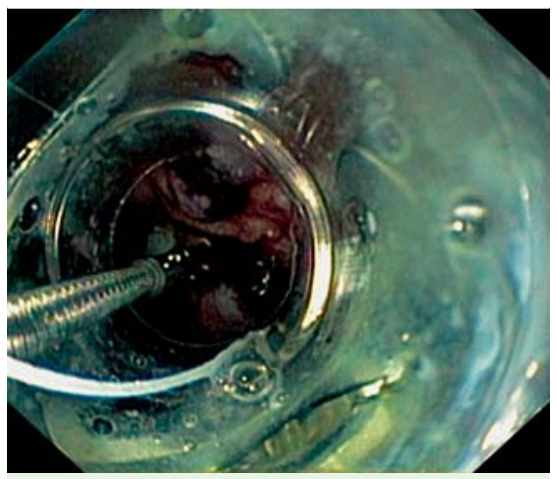

Fig. 3 The edges of the ulcer are brought together with the twin grasper and gently pulled into the application cup. The (white) firing wire passes through the working channel of the endoscope and deploys the OTSC in the same manner as in the rubber-band ligation technique.

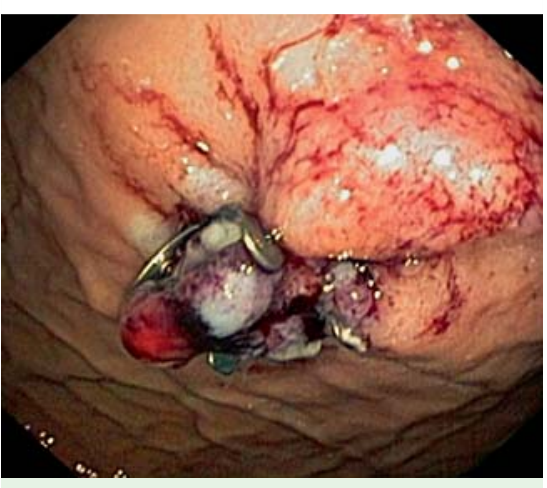

Fig. 4 The 10-mm traumatic OTSC in position.

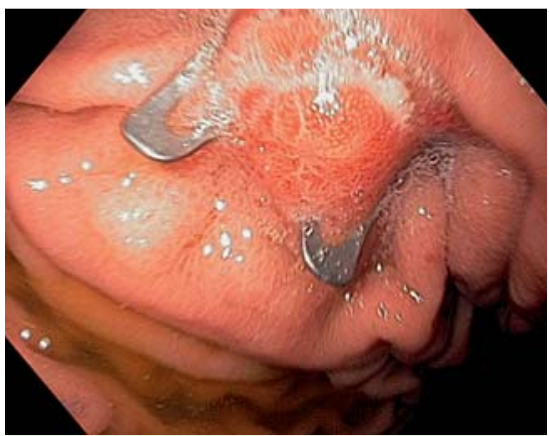

Fig. 5 The closure of the perforation is still intact 4 days after the procedure. 
Endoscopy_UCTN_Code_TTT_1AO_2AI

Endoscopy_UCTN_Code_TTT_1AO_2AN

Competing interests: None
F. Swahn, U. Arnelo, L. Enochsson, M. Löhr, T. Agustsson, K. Gustavsson, M. A. D'Souza, L. Lundell

Division of Surgery, Department of Clinical Science, Intervention and Technology (CLINTEC), Karolinska University Hospital, Karolinska Institutet, Stockholm, Sweden
Bibliography

DOI $10.1055 / \mathrm{s}-0030-1256002$

Endoscopy 2011; 43: E28-E29

(c) Georg Thieme Verlag KG Stuttgart · New York . ISSN 0013-726X

Corresponding author Fredrik Swahn, MD

Division of Surgery

Department of Clinical Science,

Intervention and Technology (CLINTEC)

Karolinska University Hospital

Huddinge

SE-141 86 Stockholm

Sweden

Fax: +46-08-58586366

fredrik.swahn@karolinska.se 